\title{
Telepresence and Tele-absence: A Phenomenology of the (In)visible Alien Online
}

"The problem, actually, does not lie in telepresence, which amplifies our own possibilities to the level where distance is abolished; but in tele-absence, which withdraws from its own access."

(Waldenfels, 2009, p. 110)

Norm Friesen, Boise State University, Boise USA

Email:normfriesen@boisestate.edu

\begin{abstract}
Proliferating media forms, from tablets to Twitter, are changing communicative practice, delimiting new experiential horizons, and thus providing phenomenological research with novel variations on the experience of self and other. Videoconferencing via Skype or FaceTime offers prominent examples of these changing forms. Despite the use of these communication technologies in both educational contexts and everyday life, educational videoconferencing has been described in the research literature as "a hidden mode of delivery, employing invisible pedagogical techniques.” In this study I address this situation of simultaneous familiarity, invisibility and uncertainty by focusing particularly on the lived experience of space, the body and eye contact in videoconferencing contexts. This study suggests that the disruption of spatial coherence and power of gaze and mutual gaze are all but unavoidable features of this experience. It concludes by emphasizing the importance forms or expressions of absence, such as the diminution of eye contact, or the importance of not always being perceived as performing or "on" in videoconferencing contexts.
\end{abstract}

\section{Introduction}

Tablets and smart phones, together with new communication services such as Skype and Twitter, have changed what it means "keep in touch." These proliferating forms are delimiting new experiential horizons, providing phenomenological research with novel variations on the experience of self and other. The experiential study of such communication is of particular importance in the fields of distance education and educational technology, disciplines which both investigate and utilize these technologies for teaching and learning. In courses and degree programs in these fields, digital media are increasingly the only means of contact between teacher and student, and between students themselves. Among these technologies, audio/video or videoconference communication is widely favored, at least in theory: "Media richness theory," as one example, "proposes that media differ in the ability to facilitate changes in understanding among communicators, [with] face-to-face communication [being] richer... than written memos because it enables immediate feedback and ...cues such as facial expressions" (Kahai \& Cooper, 2003, p. 264). The immediacy and cueing may be vital for remote students seeking advice from their teachers about their grades or their future, or for an advisor wishing to express related support 
or concern. Indeed, Microsoft's Skype, Apple’s FaceTime or Google's Hangouts boast that their users can be "in two places at once," that "conversations" can be brought "to life" or that distant interlocutors can communicate literally “face-to-face.” Freedom and immediacy are reconciled and conjoined. However, at the same time, research on video conferencing frequently brings attention to its multiple communicative challenges and educational pitfalls. Jaron Lanier, an expert on virtual realities, admits that "videoconferencing seems precisely configured to confound ... nonverbal elements [of] human interaction” (2001, p. 68). Since Lanier made this observation, videoconferencing has certainly changed in its availability, but not in its fundamental configuration.

What is the significance of these contradictory claims and realities for education? Do popular audio/visual technologies actually enable student-teacher communication that is "as good as" faceto-face? If something might be missing or "confounded" in these interactions, what might it be, how would it be experienced and how could it be addressed? Are there demands made on these technologies that are particular to education? In this paper, I respond to these questions by using hermeneutic phenomenology, the study of lived experience and its meanings, to examine and interpret incidents experienced by teachers and moderators using online conferencing technologies.

\section{The Videoconference: A well-known but hidden mode of delivery}

Given the recent and rapid rise of popular video-conferencing systems such as FaceTime and Skype, ${ }^{\mathrm{i}}$ the quantity of educational literature reporting on the effective use of audio-visual telecommunication is surprisingly small. The "body of literature on the use of videoconferencing in schools" as one recent review states, is "limited” (Lawson et al., p. 316). Another highlights "a lack of practical and field-proven models" specifically for video communication in inquiry-based learning -a finding which likely also applies more broadly. Also, over time, the practical findings and recommendations provided in the literature have changed. For example, Diane Laurillard prominently characterized videoconferencing in her 1993 Rethinking University Teaching as a "one-to-many medium, making it a sensible way to provide access for many sites to a remote academic expert" (Laurillard, p. 166). Accordingly, Lawson and Comber explain, "the use of videoconferencing in higher education institutions" initially "focused on the concept of the remote lecture” (2010, p. 296). Through further research and practice, however, researchers and practitioners have gradually come to see this medium as suited to interactive use rather than to "one-to-many" broadcasts. One 2004 review recommended that "educators should use the '15 minute rule,' limiting presentations or talking heads to no more than 15 minutes” (Greenberg, 2004, p. 17); by 2010, it is not uncommon to find studies pointing to "interaction .... as the key component of a constructivist use of videoconferencing” (Lawson et al., 2010, p. 306). However, the question remains as to how this interaction is to be realized: Interaction "in the videoconferencing medium is not without its challenges," Gilles (2008) admits -adding that while interaction is widely seen "as the key to successful videoconferencing," students engaged in such interaction have "reservations about its effective use” in actuality (pp. 110, 114).

Overall and perhaps counterintuitively, this most advanced of communication technologies seems to position the teacher in his or her most traditional role, as "a sage on the stage," rather than as a facilitator or "guide on the side" (see: King, 1993). "The learning environments that were observed... were typically teacher-centered and strongly teacher-directed," one early (1997) study reports, noting that "few teachers used instructional models that would enable them to promote higher-order learning outcomes” (Oliver \& McLaughlin, 1997, p. 51). Evidence showing that this 
pattern continues in later use and analysis is readily available. The 2008 student survey mentioned above, for example, found that students valued the "videoconference... most for the opportunities for tutor-student interaction” (Gillies, 2008, p. 115). The emphasis, evidently, was not on interaction among students but between any single student and the tutor or instructional personnel. Indeed, the videoconference not only seems to affirm the traditional responsibilities of the teacheras-sage, but actually adds to these. Besides being a lecturer and interlocutor, the teacher is often also asked be a coordinator or orchestrator -in discussion moderation (e.g., Latta \& Thompson, 2011, p. 14) or in the facilitation of appearances by remote guests and experts. Reporting specifically on the latter in the context of fine arts classes, Barniskis and Thompson (2012) explain:

This approach underscores the important role of the teacher in the room with the students, who has to function as the eyes and ears of the [guest] artist and who also can facilitate the learning in a hands on way that augments or clarifies what [the guest] is communicating. (p. 20)

This apparent contradiction between the teacher-centered realities and the interactive potential of videoconferencing is paralleled by the broader tension between its everyday familiarity and the simultaneous paucity of relevant research. As Lawson and his co-authors conclude, "the field of videoconferencing in education is... under-researched by the academic community. In many senses," they continue, "it remains a hidden mode of delivery, employing invisible pedagogical techniques" (2010, p. 307). Or as Tomlinson et al. conclude, "Videoconferencing is not new, having been used in business contexts for a number of years, but 'best practice' models for its use in education are still developing” (Falloon 2012, p. 2).

\section{Phenomenology: Bringing the Hidden to View}

In this study I address this situation of simultaneous ubiquity, invisibility and uncertainty by using phenomenological hermeneutics, a method which -despite its sometimes cumbersome nomenclature- strives to bring to attention to everyday and sometimes banal and overlooked aspects of experience. Simply put, hermeneutics is the study of "the theory and method of interpretation” (Curtis, 2004, p. 222); and phenomenology, the study of lived experience (Kenkle, 2008, p. 223). Taken together, these methodological components allow the researcher to focus on the lived meaning of an experience, with its meaning being the result of hermeneutic interpretation, and "phenomena" being the element or elements of the experience of phenomenological concern. With its focus on the unabstracted or concrete, phenomenology isolates and investigates lived experience in terms of the dimensions or "existentials" that are generally present in everyday experience: time, space, relation and the body.

Thus, in the case of the videoconference, "time" is manifest in the duration of the conference itself, and also in moment-by-moment hesitations and interruptions, whether these are part of the technical or the social dynamics of the communication. Space involves the surroundings of those connected via videoconference, and can be defined in terms of visual space and setting (e.g., lighting, staging), audible space (e.g., vocal characteristics, background noise), the participants’ literal location (at home, at work, etc.) and the positions of screens, cameras and related elements. Since it takes place between persons, videoconferencing obviously involves relation. If there is a group or a class at one of these end-points, the relations among these participants also come into 
play -and will often be compared with relations to those who are remote. The body, finally, has to do with how each presents their person in the videoconference, including how they are dressed, positioned and made (in)visible. Significantly, the body (the eyes, face and hands) is also the main source of the "non-verbal cues" that are so often highlighted as advantageous for communication via videoconference. As this paper later shows, the body also hides particular characteristics that are important for both the apparent strengths and the evident challenges of videoconferencing as an educational medium.

Besides unfolding in space and time, and being oriented to or around the body and relation, experience is also structured by "intentionality." This is not so much our explicit aims and goals, but rather the way that our awareness and consciousness is directed purposefully in and to the world around us. Thus, in a one-to-one videoconference, a participant's attention initially might be directed at the other on the screen, to communication and relation with him or her. Later, this might shift to wording in a paper, or to include discussion of other shared concerns. Particular combinations or intersections of these elements can come to represent "themes;" for example, the "theme" of "flow" and its interruption in engagement with media (Csikszentmihalyi, 1990) -and the corresponding continuity or interruption of intention in the videoconference experience. All of these experiential elements are studied by phenomenology not in technical or material terms, but as they are perceived or experienced. Thus if someone feels like a student is looking away distractedly, or that the teacher's voice is echoing as if she's in a cave, these impressions and characterizations are taken seriously and studied carefully as such. These experiences are not simply "explained away" as the result of sonic feedback or camera positioning (although these factors are certainly acknowledged).

The qualitative, experiential data in the study reported here have been gathered through a number of techniques, including "hermeneutic interviews," in which instructors who have used videoconferencing are asked to recall and describe specific incidents with students in videoconferencing settings, and to compare these experiences with possible face-to-face parallels. This paper also uses sources that are otherwise reflective of particular experiences, such as incidents described directly or indirectly in peer reviewed literature, as well as situations depicted in more vernacular sources, such as feature films and video clips. All of these are sources of possible human experience whose inclusion is intended to appeal to readers on the intersubjective level of shared experience, to be "recognizable" rather than objectively measured or statistically validated. One example of each type of evidence -a vernacular video, an incident in invoked in the "formal" literature, as well as one gleaned from a hermeneutic interview-are utilized in this paper. ii $^{\text {" }}$

The data used to study this intentionally structured experience of space, time, body and relation are presented in the form of short textual descriptions. A hermeneutic explication typically follows on such a description. This hermeneutic discussion or reflection allows for the explication of the description in terms of time, space, time, body and relation, and for reference to phenomenological writings, including those of an explicitly philosophical nature. In particular, philosophical texts from phenomenologists Sartre, Merleau-Ponty and Waldenfels, who have written with insight on perception, the body and technology, are referenced in these hermeneutic reflections. 


\section{"Oh, and one more thi...."}

This study begins with an examination of a YouTube video recently made popular in social media. As I have been planning and working on this paper, I have seen (and shared) an amusing "viral" video titled “A Conference Call in Real Life” (Crosby \& Stanton, 2014). In this case “real life” refers to the fact that the participants in the call are depicted as physically present in a typical conference room; however, all the other conditions of a conference call still hold. This type of conference is actually not a full videoconference; the participants are linked only by sound. In this sense, the video can be said to highlight intentionality, relation and space as they are mediated aurally, in isolation from visual perception. However, characteristics of this aural experience are recognizable in videoconferencing as well. (This "isolation" of aspects of an experience can be seen as illustrative of what Husserl and others after him have called "eidetic variation" in which specifically experiential "facets" are illuminated by being isolated and examined in turn. ${ }^{\text {iii) }}$ ) At the beginning of the meeting, each participant's arrival in the conference "room" is noted by the system repeating their name, and explicitly announcing their presence: “'John' has joined the conference.” Particularly in their subsequent contributions to the meeting, each participant brings with him or her the characteristics of a separate auditory environment. These include an unnatural echo, staccato or extended silences, or background noises such as a barking dog or a cappuccino machine. So although each participant is depicted as present in a shared visual space, these sounds suddenly become a part of the room's ambience when the participant speaks, and disappear just as suddenly when he or she falls silent.

However, it is the conclusion of this conference call that is my principle focus here:

As he puts his things away, the conference chair, Tripp, says: "Well thanks everyone, once again." After only the briefest pause he continues "Oh, and one more thi...." But he cuts his words short as he realizes that everyone has abruptly left. The room is now silent and empty.

This incident can be interpreted as illustrative of a phenomenon that might be called communicative “availability.” One’s openness for communication tends to be managed or signaled in highly controlled ways through digital media or technology: You pick up the ringing phone and say "hello?" You see a green highlight beside those who are logged in (with you) via Facebook or Gmail; or you hear " "John' has joined the conference” in a conference call. In each case, the possibility of communication with others tends towards a sharp "all or nothing” distinction. Others are either simply "there" or they are not. Participants are suddenly available, and just as suddenly, without transition, become unavailable -when it is too late for them to be reminded of "one more thing."

Why would the chair's (Tripp's) final moment of frustration be so implausible in a "real" face-to-face meeting? What is otherwise involved in the transition between presence and absence at a meeting like this? Unlike video or audio conferences, a face-to-face meeting of course requires people to actually make their way to a physical destination. This takes place as passage through hallways, doorways, via elevators and stairways -if not through many other pathways, conveyances and conduits. These spaces would also include, in the example above, the perimeter of the meeting room and the paths (however short) to the points of entrance and exit. Indeed, we are constantly reminded that the location independence of videoconferencing frees us from passage through any space at all. It is easy to think of these places and spaces as simply ways of getting somewhere. However, these locations can be much more than that. They are spaces of habitual 
traversal through which participants physically -and also likely mentally and socially- "converge" on their eventual purpose. After a meeting, a similar "divergence" takes place, starting from the periphery of the conferencing room, extending to the participants' "homes" or other destinations. Unlike a formal meeting-with an agenda, minutes and motions- hallways, doorways and elevators can be places for informal communication, as this convergence and divergence takes place. The hallway, doorway and elevator are all "transitional spaces” where favors may be asked or granted, important reminders given and received, and where other exchanges may take place which are literally "off the record."

Such interaction, in other words, is not predetermined by an explicit agenda. There are even genres of communication that suggest the type of communication that can take place in such spaces: The "elevator pitch," for example, refers to brief proposals made to (fortuitously encountered) customers or superiors; “water cooler chatter" -occurring neither at one's desk nor in a conference room- is seen as important for team-building, motivation and mutual understanding. Communications and management experts readily provide advice for each of these genres. It seems that it is a similar space of informal, transitional interaction that Tripp, the chair, is seeking to put to use.

From an explicitly phenomenological perspective -the perspective of possible lived experiences- these transitional places and spaces are seen to offer a wide flexibility for communication and cooperation, as Edward Casey describes:

[Such p]laces ...foster experiences that appear purposeless at first glance. We enter here an architectural realm that might be called "transitional" ... [with] such characteristics as freedom of movement (within certain definite limits) and plasticity of aim. Thus we discern the special character of indirection in built places, their nonstraightforward aspects and roundabout features [which] are much more commonly characteristic of built places than we might first imagine (2009, pp. 121-122)

The design of virtual conferencing spaces, on the other hand, tend not to have these nonstraightforward "transitional" or threshold spaces for indirection and improvisation. It is as if their design is based on the narrow formalized definition of a meeting as structured precisely by agendas, minutes, rules and orders: The meeting should begin with a review of minutes, and it ends precisely when adjourned by the chair. Participants' arrival and leave-taking are purely incidental and instantaneous, however close or far away they may actually be located from one-another geographically.

However, the transitional spaces of the hallway or elevator do not gain their improvisational, multipurpose quality all on their own. As I show in the next section, it is from the multifaceted nature of the body, in subtle combination with what is allowed in traversal and transition, that such qualities emerge. Embodiment, as I have argued elsewhere (specificallyin The Place of the Classroom and Space of the Screen, 2011) involves multiple, simultaneous and unavoidable nuances of expression of attention and commitment. One's body is always articulating a disposition and attitude, including dispositions such as passivity and receptivity. Moreover, unlike virtual presences, the body cannot be in two places at once. Online technologies, including those of videoconferencing are different, as Friesen explains:

By requiring users to log on, to click here versus there [or direct one's attention to the camera or the screen]... Web technologies repeatedly foreground explicit action over inaction. In this 
sense, online technologies simply cannot match the unavoidable force, nuance, and multiplicity of the body as an "expressive space"... [Online forms of "presence"] too often requires one to insist, for example, “I’m here for you,” while one’s actual, indivisible, indubitable embodied presence requires no such insistence. (2011, p. 158)

In videoconferencing in particular, the body is not simply erased or transcended; instead, it is foregrounded in specific and sometimes quite unexpected ways, as I describe in the next section.

\section{"Your are on-screen, and probably larger than life-size"}

My consideration of the body begins with a second piece of evidence, an incidental warning from Barniskis and Thompson (2012), whose descriptions and advice highlight the intertwining of the four experiential dimensions (body, time, space and relation) in interesting ways: "Even if... you are not 'on,"” the authors advise, "you are on-screen, and probably larger than life-size. If you surreptitiously pick your nose, chances are that everyone can see you doing it” (p. 19). Joking aside, this passage highlights the relation of "you," the teacher, to your students, or to "everyone [who] can see you;" and in their eyes, you -or rather your face and body- are "on-screen, and probably larger than life-size.” This refers to an initial characteristic of video-conferencing: its distortions of space and of the body in this space, through camera positioning and other settings (e.g., panning, zooming). We may be accustomed to seeing such "distortions" presented in conventionalized ways on TV and in movies; however, we are much less used to being thus projected and distorted ourselves, perhaps particularly in the classroom context. In his 2001 article on videoconferencing referenced above, Lanier explains this phenomenon in general terms while at the same time bringing out important relational implications: "participants aren't able to establish a sense of position relative to one another," he explains, "and [they] therefore have no clear way to direct attention, approval or disapproval” (2001, p. 68). One might also add to Lanier’s description that participants also do not know about how attention, approval or disapproval is directed toward them. This is certainly, if unconventionally, suggested in the reference to "surreptitiously pick[ing] your nose." The disquieting or comic overtones of this image point to an underlying experiential complexity: It simultaneously involves relation (being seen by others) and the body (and expectations and taboos surrounding it).

In connection with these disquieting overtones, the passage from Barniskis and Thompson is illustrative of a "theme" involving body and relation that is relatively well-developed in the phenomenological literature. This is the experience of being observed and in a way, objectified in the eyes of another. One of the earliest and perhaps most powerful accounts of such observation is provided by Jean-Paul Sartre in his famous description of "the look" (from Being and Nothingness, 1953). Sartre imagines himself in a hallway, a "transitional space,” bent or leaning, and looking through a keyhole into in an apartment -again, a surreptitious act. In this act, intention and awareness are focused on what can be seen and heard through the keyhole -being "drunk in" by what is observed "as ink is by a blotter" (p. 348). Sartre continues: "But all of a sudden I hear footsteps in the hall. Someone is looking at me!” (p. 349). A complete reversal of awareness and intentionality takes place. Instead of an intentional focus on what is on the other side of the keyhole, the protagonist's awareness is now focused on himself and his body as seen by the other: "I see myself because somebody sees me," Sartre says, "I am indeed that object which the Other is looking at and judging” (p. 349). The self, in a sense, is reduced to the embodied act (leaning and spying, or other "surreptitious" activity); and in the absence of other communication, is 
momentarily objectified in this act. The self in this context is not as much another subjectivity as it is a violator of others' reasonable expectations to privacy. As if picking up directly from Sartre's description, Merleau-Ponty explains:

The other transforms me into an object and denies me... This is what happens, for instance, when I fall under the gaze of a stranger. However, this takes place not so much in combination with other acts of communication, as in their absence... [My] objectification ... by the other's gaze is felt as unbearable only because it takes the place of possible communication. (2002, p. 420)

Of course, videoconferencing technology generally offers a number of "other acts of communication.” Indeed, through its multiple modalities, this technology can be seen to compel its users to these other communicative acts. Although its effect is perhaps heightened by the body being shown as larger than life or as otherwise distorted, the possible experience of objectifying vision is diffused in at least two ways: 1) through sound or speech, which was touched on above, and 2) mutual glance or eye contact to, a multilayered form of contact which this paper now turns.

\section{"Mutually Enfolding Glances"}

The experience of the gaze or glance in mutual eye-contact illustrates the importance of a further phenomenological theme or set of possibilities: The broader theme corresponding to this reciprocal contact is the corresponding mutuality, reversibility or reciprocity of embodied communication and contact in general. In most social settings, to be seen by another is also to see him or her. To hear another is also (at least to have the possibility) to be heard; to touch is also to be felt (as ritualized in the handshake). To be approached by another, who for example asks for the time perhaps glancing at one's eyes and also at one's wrist- normally has little that is unbearable or distressing about it, unlike the look in Sartre's hallway. This perceptual openness, sharing and reciprocity is perhaps at its most intensive in mutual eye-contact, as Merleau-Ponty recognizes:

I look at him. He sees that I look at him. I see that he sees it. He sees that I see that he sees it... Well, even though in principle reflections upon reflections go on to infinity, vision is such that the obscure results of two glances adjust to each other, and there are no longer two consciousnesses with their own teleology but two mutually enfolding glances. (1964, p. 17)

In even the briefest moment of eye-contact, as one psychologist puts it, "the other is attending to your attention while you are attending to hers” (Stawarska, 2006, p. 19). In thus seeing and being seen (as seeing), as Merleau-Ponty suggests, a kind of perceptual alignment is achieved. This alignment extends to the awareness of both involved, and even to their intentional focus or aim (teleology). In everyday expression, to say something while looking another "in the eye" is to "really mean" what has been said, and to presume to have been heard and believed. At the same time, eye-contact has freighted and highly ambivalent social meanings: It can prompt the aggressive "who're you looking at?", or be accompanied by the affirmative "here's looking at you," or it can lie somewhere in-between: "s/he gave me the look," a comment regarding a communicative act that can have any number of specific meanings. Again, in most social settings, we believe we can feel that the gaze of another upon us, as if it has an almost tactile force. 
Corresponding to this significance, there is no shortage of quantitative evidence on the value of eye-contact in collaboration and instruction.

For example, mutual gaze is associated with increased cognitive task performance (Dalzel-Job, Oberlander, \& Smith, 2011), and Bailenson, Beal, and Blascovich (2002) emphasize the importance of mutually confirmed gaze in teaching:

During instruction, gaze helps learning, in that college students had higher performance on a learning task when the instructor gazed at them than when the instructor did not. Furthermore, when students are able to return the gaze to the instructor, they participate more in the instruction than when they [cannot]. (p. 315) ${ }^{\text {iv }}$

However, this type of reciprocal contact is broken or fractured in the videoconference. The necessarily different positions of the camera and screen in videoconferencing systems leads to a disruption of precisely this "mutually enfolding" potential of the gaze. Eye-contact of a kind can occur, but in such systems, it cannot be simultaneously reciprocal. You cannot truly look an interlocutor in the eye since seeing another's eyes means looking at the screen. You can give the appearance of making eye-contact, but this actually requires looking away from the other, and into the camera, generally positioned above the screen. This problem was recognized and studied by Bell Labs in the design of the first "Picturephone" (Stokes, 1969). Beginning at this early stage and since, attempts have since been made to address this problem through sophisticated combinations of software and camera hardware, or teleprompter-like configurations of screen, camera and bidirectional mirror(s). On the other hand, some software designs exacerbate the difficulty by placing the images of one's interlocutors not at the top but at the bottom of the screen, often the furthest distance from the video camera. It is precisely this configuration that forms the basis for the final piece of experiential evidence to be examined in this paper. This brief description is derived from an interview of an instructor who regularly meets with multiple students over videoconference:

When I'm videoconferencing with my class, I always want to multitask -to check my email, hiding the row of student faces at the bottom of the screen. But then I just feel guilty: they think I'm looking at them, at the top of the screen, when I'm actually doing something else entirely! (Lisa, interviewee $)^{v}$

Underlying this description is a singular experiential situation: One in which the instructor gives her students the impression that she is looking at them squarely in the eye precisely when she is not. This effect may be said to be curiously multiplied, because the instructor is not looking at a single student, but at multiple "pairs of eyes" all at the same time. On the face of it, this is not too different from a newscaster or politician broadcasting via a teleprompter. However, while the scripting, display and timing of a teleprompter imply a rather elaborate set up to achieve an intended effect, in the case of the instructor, such apparent eye-contact or its absence actually happens by accident. 


\section{Conclusion: Telepresence or Tele-absence?}

As this description suggests, eye contact is about much more than one body or "self" seeing another, and the other simply reciprocating. It is instead a multidimensional form of communication of its own, with multiple layers or moments of perceptivity and receptivity -as descriptions from phenomenology and psychology suggest. Something similar can be said for the appearance of one's person of body in the videoconference, and for the "virtual" visual and acoustic space constituted by this medium: there is more to each of them than initially meets the eye (or ear). We expect of this virtual space more flexibility (or "nonstraightforwardness") than what is suggested by the most basic components of a meeting. And we may be surprised by the way we feel (or do not feel) ourselves to be observed, and by the ways the body may signal various -and not always desirable- functions and meanings while in this context. Given the multifaceted nature of the examples and other evidence considered here, some immediate and pragmatic implications can be developed. Three of these implications -corresponding to the three principle pieces of evidence examined- can be summarized as follows:

1. The description of the adjournment of the teleconference highlights the importance of transitional spaces. These spaces cannot, of course, be literally or fully reconstituted in mediated contexts, but their absence might be compensated for through the use of other communication forms that register communicative availability in various ways, including the asynchronicity of email and of text messages or Twitter. These last two forms, text and Twitter, may be particularly helpful, since they are closer to real-time communication than email, but otherwise more flexible and tolerant of communicative "gaps," such as the pause between Tripp’s initial farewell and his subsequent announcement or reminder.

2. The warning about being "always on and probably larger than life" underscores the objectification of one's appearance in the unblinking eye of the camera and the simultaneous lack of awareness of this surveillance. The investigation of this situation points to the importance of the absence of "other acts of communication" (Merleau-Ponty, 2002, p. 420) in connection with such objectification. This analysis suggests one possible solution, which would be to deliberately eliminate visual contact when other communicative acts are not taking place. This would involve not more and better technology, but a discrete attenuation of its intrusion and default operation. Voice and vision, in other words, should broadly coincide. In the words of Barniskis and Thompson (2012), this would mean making it easy or automatic to actually not be "on" when salient communication is occurring elsewhere.

3. The third example highlights the value of expanding the range of "other acts of communication" in videoconferencing to include modes of mutual eye-contact. The experiential importance of this eye-contact, as well as the importance of its apparent and mistaken presence in the videoconference points to the value of actively facilitating such contact in videoconferencing. This further suggests that using devices in two-way communication that can overcome this difficulty (e.g., by using teleprompter-like arrangements of screen and bi-directional mirrors) may be worthwhile. ${ }^{\mathrm{vi}}$ It is important to note that such a device would have to be used by all participants equally in video communication, since its function is clearly mutual or reciprocal in nature. 
To draw its philosophical or theoretical deliberations to their own conclusion, this paper briefly returns to the multiple reflexivity of sight and of the body overall, particularly as this reflexivity is understood by one of Merleau-Ponty's most prominent students, Bernhard Waldenfels. Waldenfels builds on Merleau-Ponty's explanations by saying that the body as a whole does not conform to any non-reflexive dualisms or bifurcations of subject/object or perceiver/perceived:

our body does not fit into this dualistic scheme. On the one hand, our body is exactly both at once: seeing and seen, hearing and heard, touching and touched, moving and moved. This ...characterizes the very being of our body, which refers to itself and at the same time evades itself. (2011, p. 49)

Despite -or perhaps because of- its many reflexive senses and its multiple potentialities, forms and functions, the body in a sense escapes from and exceeds itself. As Merleau-Ponty puts it, "the experience of our body... reveals an ambiguous mode of existing.... It is always something other than what it is...” (1962, pp. 230-231). ${ }^{\text {vii }}$ The experience of our own bodies, in other words, is ambiguous and protean, exceeding any momentary action, expression, perception or impression. Perhaps one of the most obvious examples of these characteristics -as it is manifest in multimedia communication - is that we do not see ourselves as others see us, nor do we hear ourselves as we are heard:

When I hear my voice on a tape or see my face in a video I get into a situation where proximity and distance are entangled, and where all direct reflection is diverted by a peculiar form of deflection. (Waldenfels 2011, p. 49)

The tone of our words and the situation of our bodies in the world are never fully available to our own perception, awareness and control. Videoconferencing contexts only seem to heighten this tendency to excess and escape, as the incidents of the conference-call adjournment and of being "on-screen...larger than life" illustrate. The body manifests presence and absence, visibility and invisiblity in ways more complex than the initial expectations in these scenarios seem to allow. A similar but more complex and perhaps ambivalent tendency towards this effect is also illustrated by the discussion of eye-contact, discussed above. We feel ourselves to be observed, and often believe our own gaze to be felt by those upon whom it momentarily rests. Whether the focus is on the eyes, the face or on a person taking his leave, the expressive and receptive space of the body is alternatively concealed and exaggerated in ways of which we cannot be fully aware, particularly when mediated through technologies such as video-conferencing.

Waldenfels characterizes that which thus escapes our awareness and control -or as he says that which "withdraws" from us- as ultimately being "alien” to us. By this he means that it cannot or does not fit into the "order" that constitutes our self-awareness, or our "sphere of ownness," as he puts it. (See the review essay on Waldenfels in this issue, pp. 68-77, for more). As the findings of this paper have shown, this order of the self is one that is disrupted and distorted by technical mediation, as Waldenfels himself acknowledges:

Technology, which has for a very long time -despite all the contemporary lamentations about alienation through technology- been regarded as an extension of the sphere of ownness, now proves more and more to be a source of alienation of its own kind. (2011, p. 6) 
Videoconferencing in this sense is also not simply an extension of the self and its order into other, remote realms, but is its own source of disruption and distortion of self and body. It is a "multiplier" of the alien in this sense. Moreover, Waldenfels predicts that minimizing or eliminating this intensified experience of the alien will be impossible, although he predicts that this task of minimization will stand as the principal challenge for technologies of telepresence such as videoconferencing. His prediction opens with the quote provided at the outset of this paper:

What would a medium capable of mediating the immediate look like? [...] The problem, actually, does not lie in telepresence, which amplifies our own possibilities to the level where distance is abolished; but in tele-absence, which withdraws from its own access. The withdrawal of the alien, which is also entrenched in our perception, strikes me with more force than the resistance of the alien, which is something I can defend myself against. With the latter, it is only a question of possessing greater or lesser force. But this is not the case for withdrawal, which is like a shadow that cannot be grasped... All technical artifice runs up against an inner border: If the alien were there, it would not be what it is. Even a video camera, which can not only register our voice and breathing, but even the lifting of the eyelids or the creasing of the brow, would fail when it comes to the glance that is more than something that is seen [or recorded], or to the voice that is more than something that is heard [or taped] -because voice and glance disrupt, incite, interrupt. Here technical media run up against the limit of representability, without being able to represent this limit themselves ... (2009, pp. 110-111)

As a technology of telepresence, video does not allow us to be present to ourselves, for example, by seeing one's own gaze or hearing one's “real” voice. Instead, the means by which we are present and absent to ourselves and to others are both augmented and distorted, amplified and attenuated: One might hear oneself on the other end of the line as a faint echo in a Skype call, for example. However, this is neither what one's remote interlocutors hear, nor how you hear yourself. A student might also withdraw his or her gaze in a moment of discomfort or reticence. However, without the ability to register the positive mutual contact of the gaze in the first place, a moment of withdrawal, as Waldenfels says, "would withdraw from its own access." As a retreat of an alien aspect of another's body, "it would not be what it is” when thus mediated. Deprived of its ability to disrupt, incite or interrupt, the glance -particularly in its withdrawal- is an example of something standing beyond the unrepresentable limit of representability common to all communication technologies.

In this sense, Waldenfels responds to his own question by insisting that it ultimately can't be answered: "What would a medium capable of mediating the immediate look like?" Such an ideal form of telepresence would be impossible, just as any full or immediate self-presence -e.g., seeing or hearing oneself as others do and also while others do- is also impossible. At best, the gaze and voices of participants in a videoconference are reflected and refracted as if in a rudimentary hall of mirrors and echo chamber, with their coincidence being at times as much unintentional or illusory as directly expressive or intentional.

\footnotetext{
i Skype alone has over 300 million subscribers.

ii Although it is not used here, scenes from the 2009 feature film Up in the Air could also readily be employed in this study. It tells the story of a travelling businessman (played by George Clooney) who is grounded by the "virtualization" of his off-site meetings through videoconferencing.
} 
iii Husserl (1970) explains this technique by referring to the use of the researcher's imagination and the example of a physical table: "Let us make this clear to ourselves, and then fruitful for our method. Starting from this tableperception as an example, we vary the perceptual object, table, with a completely free optionalness, yet in such a manner that we keep perception fixed as perception of something, no matter what. Perhaps we begin by fictively changing the shape or the color of the object quite arbitrarily, keeping identical only its perceptual appearing. In other words: Abstaining from acceptance of its being, we change the fact of this perception into a pure possibility, one among other quite "optional" pure possibilities - but possibilities that are possible perceptions. We, so to speak, shift the actual perception into the realm of non-actualities, the realm of the as-if, which supplies us with "pure" possibilities, pure of everything that restricts to this fact or to any fact whatever" (pp. 70-71). Today, this exploration of actualities and non-actualities need not understood as pure, imagined possibility; but can be actively explored using different virtual technologies and modalities that isolate and combine sound, vision, color and structure.

iv Elsewhere in the Phenomenology of Perception, Merleau-Ponty is ambivalent about the results of mediation on such a convergence of attention and intention: "And if someone does communicate with someone else, if the interworld is not an inconceivable in-itself and must exist for both of us, then again communication breaks down, and each of us operates in his own private world like two players playing on two chessboards a hundred miles apart. But here the players can still make known their moves to each other by telephone or correspondence, which means that they are in fact participants in the same world.” (2002, p. 416)

$v$ The interviewee is identified with a pseudonym.

${ }^{\text {vi }}$ One such device is the See Eye 2 Eye - webcam teleprompter \& eye contact device; see: http://www.bodelin.com/se2e

vii The full passage from Phenomenology of Perception which these characterizations are taken can be productively compared to Waldenfels' remarks: “The experience of our own body, on the other hand, reveals to us an ambiguous mode of existing. If I try to think of it as a cluster of third person processes_- sight', 'motility', 'sexuality'-I observe that these 'functions' cannot be interrelated, and related to the external world, by causal connections, they are all obscurely drawn together and mutually implied in a unique drama. Therefore the body is not an object. For the same reason, my awareness of it is not a thought, that is to say, I cannot take it to pieces and reform it to make a clear idea. Its unity is always implicit and vague. It is always something other than what it is, always sexuality and at the same time freedom, rooted in nature at the very moment when it is transformed by cultural influences, never hermetically sealed and never left behind” (pp. 230-231).

\section{References:}

Bailenson, J.N., Beall, A.C., \& Blascovich, J. (2002). Mutual gaze and task performance in shared virtual environments. Journal of Visualization and Computer Animation, 13, 1-8.

Barlow, J.P. (1996). A declaration of the independence of cyberspace. Retrieved from: https://projects.eff.org/ barlow/Declaration-Final.html

Barniskis, B. \& Thompson, L.M. (2012). The art of the videoconference lesson: Practical application and implications. Teaching Artist Journal 10(1), 15-23.

Casey, E. (2009). Getting back into place, second edition: Toward a renewed understanding of the place-world. Bloomington: Indiana University Press.

Crosby, T. \& Stanton, T. (2014). A teleconference in real life. Retrieved from http://www.youtube.com/watch?v=DYu_bGbZiiQ

Csikszentmihalyi, M. (1990). Flow: The psychology of optimal experience. New York: Harper.

Curtis, S. (2004). Still/moving: Digital imaging and medical hermeneutics. In L. Rabinovitz \& A. Geil Memory Bytes (Eds.), History, technology, and digital culture (pages of chapter). Durham NC: Duke University Press. 
Dalzel-Job, Oberlander, \& Smith, T.J. (2011). Don't look now: The relationship between mutual gaze, task performance and staring in Second Life. Proceedings of the 33rd Annual Conference of the Cognitive Science Society. 832-837.

Friesen, N. (2011). The place of the classroom and the space of the screen. New York: Peter Lang.

Gillies, D. (2008). Student perspectives on videoconferencing in teacher education at a distance Distance Education 29(1), 107-118.

Greenberg, A. (2004). Navigating the sea of research on video conferencing-based distance education: A platform for understanding research into the technology's effectiveness and value. Pleasanton, CA: Polycom.

Falloon, G. (2012). Using videoconferencing in a school-scientist partnership: students' perceptions and scientists’ challenges. Research in Learning Technologies 20(1) 1-18. http://www.researchinlearningtechnology.net/index.php/rlt/article/view/17194/html

Husserl, E. (1970). Cartesian meditations. The Hague: Martinus Nijhoff.

Kahai, S.S. \& Cooper, R.B. (2003). Journal of Management Information Systems / Summer 2003, 20(1), pp. 263-29

King, A. (1993). From sage on the stage to guide on the side. College Teaching (41)1 30-35.

Klenke, K. (2008). Qualitative research in the study of leadership. Bingley, UK: Emerald Publishing Group.

Lawson, T. \& Comber, C., Videoconferencing in English schools: one technology, many pedagogies? Technology, Pedagogy and Education. 19(3), 315-326.

Lawson, T., Comber, C., Gage, J., \& Cullum-Hanshaw, A. (2010). Images of the future for education? Videoconferencing: a literature review. Technology, Pedagogy and Education 19(3), 295-314.

Lanier, J (2001). Virtually there: Three dimensional teleimmersion may eventually bring the world to your desk. Scientific American 284(4): 66-75.

Laurillard, D. (1993). Rethinking university teaching: A Framework for the Effective Use of Educational Technology. Routledge, London.

Merleau-Ponty, M. (1964). Signs. Evanston: Northwestern University Press.

Merleau-Ponty, M. (1962) Phenomenology of perception. London: Routledge.

Oliver, R. \& McLoughlin (1997).Interactions in audiographics teaching and learning environments. American Journal of Distance Education 11(1), 34-54.

Sartre, J.-P. (1956). Being and nothingness. New York: Washington Square Press.

Stawarska, B. (2006). Mutual gaze and social cognition. Phenomenology and the Cognitive Sciences 5: 17-30.

Stokes, R. (1969). Human factors and appearance design considerations of the Mod II Picturephone Station Set. IEEE Transactions on Communication Technology 318-323. 
Tomlinson, J. Shaw, T., Munro, A., Johnson, R., Madden, D.L., Phillips, R., \& McGregor, D. (2013). How does tele-learning compare with other forms of education delivery? A systematic review of tele-learning educational outcomes for health professionals. NSW Public Health Bulletin (2) 70-75.

Waldenfels, B. (2011). Phenomenology of the alien: Basic concepts. Evanston: Northwestern University Press.

Waldenfels, B. (2009). Ortsverscheibungen, Zeitverscheibungen: Modi leibhafter Erfahrung. Frankfurt: Suhrkamp. 